\title{
A Semantic Platform for Surveillance of Adverse Childhood Experiences
}

\section{Jon Hael Simon Brenas, Eun Kyong Shin, Arash Shaban-Nejad}

UTHSC/ORNL, Memphis, Tennessee, United States

Objective

We introduce the Semantic Platform for Adverse Childhood Experiences (ACEs) Surveillance (SPACES). It facilitates the access to the relevant integrated information, enables discovering the causality pathways and assists researchers, clinicians, public health practitioners, social workers, and health organization in studying the ACEs, identifying the trends, as well as planning and implementing preventive and therapeutic strategies.

\section{Introduction}

Adverse Childhood Experiences (ACEs) have been linked to a variety of detrimental health and social outcomes. In the last 20 years, the association between ACEs with several adult health risk behaviors, conditions, and diseases including suicides, and substance abuse [1], mental health disturbances and impaired memory [2], nervous, endocrine and immune systems impairments [3], and criminal activities [4] have been studied. One of the challenges in studying and timely diagnosis of ACEs is that the links between specific childhood experiences and their health outcomes are not totally clear. Similarly, an integrated dataset buil tfrom multiple sources is often required for effective ACEs surveillance. The SPACES project aims at providing a semantic infrastructure to facilitate data sharing and integration and answer causal queries [5] to improve ACEs surveillance.

\section{Methods}

We create the ACEs ontology to facilitate the integration of data coming from various distributed sources (e.g. ontologies, databases, surveys, interviews, and literature) and maintain interoperability between the data sources. We re-used some of the existing bioontologies in the domain, although they captured the domain's knowledge in different levels of granularity, e.g., Homeless is defined in SNOMED CT and Medical Dictionary for Regulatory Activities (MedDRA) with different hierarchies, or some related concepts may be disconnected, e.g., SNOMED CT defines Abuse but not Verbal Abuse while MedDRA defines Verbal abuse but not as an Abuse. In order to improve reasoning with the aggregated data, we perform two different kinds of inference. Semantic inference uses the ACEs ontology to creates new conclusions by connecting similar concepts. On the other hand, statistical inference is used to formulate rules that link co-occurring concepts. These two kinds of inference, statistical and semantic, work in tandem and the conclusions from one method can then be used as the basis for the other kind of inference.

\section{Results}

The ACEs Ontology is a unique resource for capturing knowledge in the domain of Adverse Childhood Experiences. The ontology will be openly and freely available via the relevant online community's portals (e.g. The NCBO BioPortal). The logical validation of the ontology is performed using description logic reasoners. A set of use-case scenarios are designed to demonstrate the feasibility and usability of the ontology for data integration and intelligent query answering.

\section{Conclusions}

In this paper, we present the SPACE project that aims to develop a platform to improve adverse childhood experiences surveillance. The tool uses semantic and statistical methods to improve data access, integration, and reasoning.

\section{Acknowledgement}

This work was funded by Memphis Research Consortium (MRC). 


\section{References}

1. Felitti VJ, Anda RF, Nordenberg D, Williamson DF, Spitz AM, et al. 1998. Relationship of childhood abuse and household dysfunction to many of the leading causes of death in adults: The adverse childhood experiences (ace) study. Am J Prev Med. 14(4), 245-58. PubMed https://doi.org/10.1016/S0749$\underline{3797(98) 00017-8}$

2. Anda RF, Felitti VJ, Bremner JD, et al. 2006. The enduring effects of abuse and related adverse experiences in childhood: A convergence of evidence from neurobiology and epidemiology. Eur Arch Psychiatry Clin Neurosci. 256(3), 174-86. PubMed https://doi.org/10.1007/s00406-005-0624-4

3. Danese A, McEwen BS. 2012. Adverse childhood experiences, allostasis, allostatic load, and age-related disease [Allostasis and Allostatic Load.]. Physiol Behav. 106(1), 29-39. PubMed https://doi.org/10.1016/j.physbeh.2011.08.019

4. Garbarino J. 2017. Aces in the criminal justice system [Child Well-Being and Adverse Childhood Experiences in the US.]. Acad Pediatr. 17(7) (Supplement), S32-33. PubMed https://doi.org/10.1016/j.acap.2016.09.003

5. Okhmatovskaia A, Shaban-Nejad A, Lavigne M, Buckeridge DL. 2014. Addressing the challenge of encoding causal epidemiological knowledge in formal ontologies: a practical perspective. Stud Health Technol Inform. 205, 1125-29. PubMed 